\title{
Differential effects of alpha-adrenoceptor blockade on essential, physiological and isoprenaline-induced tremor: evidence for a central origin of essential tremor
}

\author{
B ABILA, JF WILSON, RW MARSHALL, A RICHENS
}

From the Department of Pharmacology and Therapeutics, University of Wales College of Medicine, Cardiff $U K$

SUMMARY Intravenous thymoxamine reduced the power of essential tremor but increased that of physiological and isoprenaline-induced tremor. These findings indicate that essential and physiological tremor have dissimilar pathophysiological mechanisms. They also suggest that central adrenergic mechanisms are involved in the pathophysiology of essential tremor and that isoprenaline-induced tremor is not a good model of essential tremor. Furthermore, alphaadrenoceptor blockers may be a useful therapy for essential tremor.

Several studies have shown that normal postural tremor, commonly known as physiological tremor, is caused mainly by oscillations arising from instability in the gamma loop servomechanism of the stretch reflex arc. ${ }^{1-3}$ Isoprenaline and other $\beta$-adrenoceptor agonists stimulate tremorogenic $\beta 2$-adrenoceptors in skeletal muscle causing an enhancement of this physiological tremor. ${ }^{4-6}$ By comparison, the pathophysiology of essential tremor has been less well studied and its origin and mechanism remain uncertain.

The similarity in the peak frequency of physiological and essential tremor, and the fact that age, anxiety and emotional upset affect both tremors in similar ways led to the belief that essential tremor is an exaggerated form of physiological tremor. ${ }^{7}$ In support of a peripheral origin for essential tremor, Warren et $\mathrm{l}^{\mathrm{s}}$ reported that catecholamine levels in a group of essential tremor patients were higher than those in normal controls. On the other hand, the finding that alcohol reduced essential tremor when given orally but had no effect by the intra-arterial route $^{y}$ suggests that the tremor arises from an abnormality within the central nervous system.

Address for reprint requests: Dr JF Wilson, Department of Pharmacology and Therapeutics, University of Wales College of Medicine, Heath Park, Cardiff CF4 4XN, UK.

Received 9 November 1984 and in revised form 15 February 1985. Accepted 2 March 1985.
In $1981 \mathrm{Mai}$ and Olsen ${ }^{10}$ reported that a single dose of the specific alpha-adrenoceptor blocking drug, thymoxamine, reduced essential tremor but had no effect on Parkinsonian tremor. There is evidence from studies in animals and man, that thymoxamine acts centrally, depressing gamma motor neuron activity within the spinal cord. ${ }^{11-13}$ The present studies re-investigated the effect of thymoxamine on essential tremor and assessed its effect on physiological and isoprenaline-induced tremors. It was thought that such comparative studies might yield further information on the pathophysiology of essential tremor.

Patients, subjects and methods

Two studies were performed. In the first, the effects of intravenous thymoxamine and placebo on tremor, blood pressure and heart rate in essential tremor patients were studied. In the second, the effects of the same treatments on physiological and isoprenaline-induced tremor were investigated in healthy volunteers. These studies were approved by the South Glamorgan Health Authority (T), Division of Medicine Ethics Committee before they were commenced.

\section{STUDY 1: ESSENTIAL TREMOR}

Patients, drugs and doses

Six patients (four male) with benign essential tremor of varying severity and duration participated in this study after giving informed voluntary written consent. They were aged from 38 to 69 (mean 51.6) years and weighed be- 
tween 51 and 80 (mean 65.2) kg. Apart from their tremor, all patients were in good health as judged from pre-trial clinical assessment and laboratory tests which included full blood count, liver function tests, and serum electrolyte and urea estimations. Routine drug therapy was withdrawn two weeks before the study commenced. Two patients failed to complete the study for social reasons, each missing the last treatment which turned out to be the higher dose of thymoxamine.

Two intravenous doses of thymoxamine (Opilon injection, Warner) were given to each patient: $100 \mu \mathrm{g} / \mathrm{kg}$ (T1) and $150 \mu \mathrm{g} / \mathrm{kg}$ (T2). The placebo was sterile $0.9 \% \mathrm{NaCl}$ solution for injection.

\section{Methods}

Each patient received, at 3 day intervals, placebo or one of the two doses of thymoxamine in a randomised doubleblind cross-over design. The daily procedure was as follows. After a 30 minute rest period, pre-treatment tremor, blood pressure and heart rate were recorded. The injection, which was made up to the same volume for each patient with sterile $0.9 \%$ saline, was then given over a 3 minute period into an antecubital vein in the dominant arm. Tremor, blood pressure and heart rate measurements were repeated immediately after the injection, then every $15 \mathrm{~min}$ for 1 hour.

Blood pressure was estimated with a Hawksley random-zero sphygmomanometer while heart rate was measured by electrocardiography. Hand tremor was quantified by means of a microcomputer based accelerometric technique using a subminiature piezoresistive accelerometer taped to the dorsum of the middle finger of the non-dominant hand (that is the left hand in a righthanded subject). ${ }^{14}$ For each recording, the patient sat upright with the arm supported up to the wrist and the hand was held in the horizontal position for 20 seconds. Tremor was recorded during the last 10 seconds and the hand was allowed to rest between recordings, which numbered five for each assessment. Each 10-second tremor record so collected was filtered to remove high frequency noise, digitised at a sampling rate of $50 \mathrm{~Hz}$ and subjected to power spectral analysis.

The spectral analysis method used Fourier transformation of the autocovariance function and incorporated a Bartlett smoothing function with a lag window of 120 for the $\mathbf{5 0 0}$ data points analysed. The value used for subsequent analyses was the total tremor power between 2 and $14 \mathrm{~Hz}$, hereafter referred to as tremor power. Tremor power below $2 \mathrm{~Hz}$ was omitted in order to eliminate any contribution made by gross changes in position of the hand which were unrelated to tremor. There were no significant power components in the spectrum above $14 \mathrm{~Hz}$.

\section{STUDY 2: PHYSIOLOGICAL AND} ISOPRENALINE-INDUCED TREMOR

\section{Subjects and drug doses}

This study was in two sections. Section A was concerned with basal physiological tremor and section B with isoprenaline-enhanced physiological tremor, termed isoprenaline-induced tremor. Eight healthy, drug-free, non-smoking males aged 19-30 (mean 24.5) years and weighing between 57 and 83 (mean 67.2) kg participated in both sections of this study after giving informed voluntary written consent.

Placebo and thymoxamine (T1 and T2) injections were the same as those in Study 1. Isoprenaline, used in section B of this study, was prepared by the hospital pharmacy. Ampoules contained $5 \mathrm{ml}$ of $1 \mathrm{mg} / \mathrm{ml}$ isoprenaline sulphate in water with $0.1 \%$ sodium metabisulphite as an antioxidant. For infusion, the calculated amount was made up to $60 \mathrm{ml}$ with $5 \%$ dextrose in a plastic syringe and delivered into an antecubital vein in the subject's dominant arm using a servocontrol infusion pump (Harvard Apparatus).

\section{Methods}

The experimental design and methods used in section A were the same as for Study 1 on essential tremor patients.

In section $B$, preliminary trials were performed to determine an isoprenaline infusion rate for each subject that would increase tremor by $100 \%$ or more, without causing a tachycardia of more than 30 beats per min. These dose rates ranged from 1.0 to $2.5 \mu \mathrm{g} / \mathrm{min}$. The accelerometer for measurement of tremor and ECG limb leads were connected and the subject, who remained supine throughout the procedures, rested for $\mathbf{3 0}$ minutes. Pre-treatment tremor and heart rate were recorded and the isoprenaline infusion was then started at the selected dose rate and continued for $90 \mathrm{~min}$. Measurements of isoprenalineenhanced heart rate and tremor were taken between the 20 th and 25 th min of the infusion, by which time preliminary studies showed that both heart rate and tremor had reached steady state levels. The thymoxamine or placebo was then injected over a 3 min period. Measurements were repeated immediately after the injection, then every 15 min for $1 \mathrm{~h}$. Blood pressure was not estimated during this section of the study.

As in Study 1, placebo and the two doses of thymoxamine were given in a randomised, double-blind, crossover design to each subject, treatments being at 3-day intervals.

\section{Data analysis}

Tremor power was log-normally distributed, hence the logarithmically transformed values were used for analysis. Individual subject and patient data for each parameter measured were subjected to 3-way analysis of variance by treatment, time and subject. Significant differences between means were identified by the least-significant difference approach. Percentage reductions or increases in tremor given in the text were calculated from the logarithmically transformed values.

\section{Results}

\section{Blood pressure and heart rate}

Changes in heart rate and diastolic blood pressure following the treatments in Studies 1 and $2 \mathrm{~A}$ are shown in table 1 . There were no significant changes following treatment with placebo. Both doses of thymoxamine caused significant decreases in diastolic blood pressure and increases in heart rate. 
Table 1 Blood pressure and heart rate measurements before and after treatment with thymoxamine or placebo in healthy subjects and patients with essential tremor.

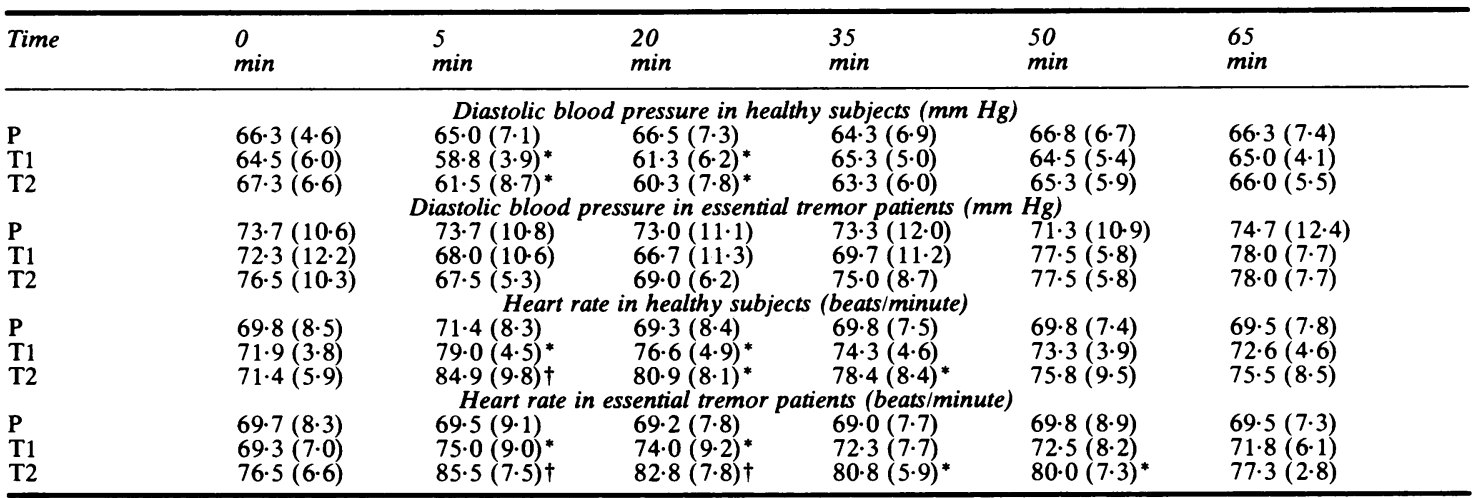

${ }^{*}$ Significantly different from corresponding placebo values:

$\mathrm{p}<0.05$, t $\mathrm{p}<0.01$.

0 min = pre-treatment values; 5 min- $65 \mathrm{~min}=$ values after injection. Values are expressed as mean (SD). $\mathrm{N}=6$ except for T2 treatment of essential tremor patients $(\mathrm{N}=4)$. $\mathrm{P}=$ placebo; $\mathrm{T} 1=$ thymoxamine $100 \mu \mathrm{g} / \mathrm{kg}$ i.v.; $\mathrm{T} 2=$ thymoxamine $150 \mu \mathrm{g} / \mathrm{kg}$ i.v.

Changes in systolic blood pressure paralleled the changes in diastolic blood pressure.

\section{Tremor frequency}

None of the treatments had any significant effect on the peak frequency of tremor in healthy subjects or patients; nor was the peak frequency of isoprenaline-induced tremor affected ( $p>0 \cdot 1)$. Any further mention of tremor refers to tremor power.

\section{Tremor power}

\section{(1) Intersubject variability}

In accord with published data, significant intersubject variations in tremor were observed for all three types of tremor $(p<0.001)$. The range of mean pretreatment tremor power was 140 to $21056 \mathrm{mg}^{2} / \mathrm{s}$ for essential tremor, 10.8 to $74.3 \mathrm{mg}^{2} / \mathrm{s}$ for physiological tremor and 47.3 to $162.0 \mathrm{mg}^{2} / \mathrm{s}$ for isoprenaline-induced tremor.

(2) Drug effects

Placebo treatment produced no significant change $(p$ $>0.2$ ) in basal physiological, isoprenaline-enhanced physiological or essential tremor (figs 1 to 3 ). The maximum observed changes for the three types of tremor were respectively $-12.4 \%,-16.1 \%$ and $+34.6 \%$ in absolute units or $-4.1 \%,-3.9 \%$ and $+4.4 \%$ in logarithmic units.

Basal physiological tremor was significantly increased after administration of $T 1$ and $T 2$, the maximum increases being recorded 5 minutes after injection (fig 1). The increase after T1 was $25.8 \%$ in absolute units or $6.9 \%$ in logarithmic units, while that after T2 was similarly $39.8 \%$ or $10.4 \%$. The tremor values at this point, and at 20 minutes, were

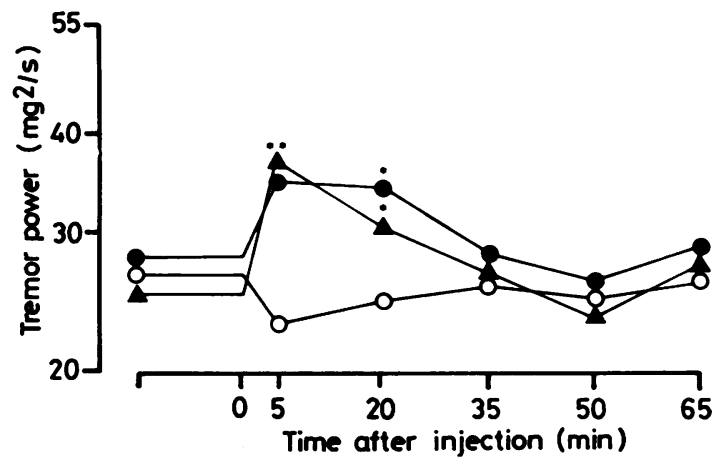

Fig 1 Effects of intravenous thymoxamine or placebo on the power of basal physiological hand tremor; (O) placebo; (O) thymoxamine $0.1 \mathrm{mg} / \mathrm{kg}$; (A) thymoxamine 0.15 $\mathrm{mg} / \mathrm{kg}$. Each point is the mean from 8 subjects. Points significantly different from the corresponding placebo value are marked ${ }^{*}(p<0.05)$ and ${ }^{* *}(p<0.01)$.

significantly different from the corresponding values after placebo. There was no significant difference between the effects of $\mathrm{T} 1$ and $\mathrm{T} 2$ at any time point $(p>0.05)$.

Isoprenaline-enhanced physiological tremor was also significantly increased by thymoxamine (fig 2 ). The maximum increase after $\mathrm{T} 1$ was $22.0 \%$ in absolute units or $4.6 \%$ in logarithmic units, while that after T2 was $22.2 \%$ in absolute units or $4.3 \%$ in logarithmic units. The effects of $\mathrm{T} 1$ and $\mathrm{T} 2$ were significantly different at 20 and $35 \mathrm{~min}(\mathrm{p}<0.05)$.

Both $T 1$ and $T 2$ produced significant reductions in essential tremor which reached a maximum at 20 min (fig 3). The maximum reduction after $\mathrm{T} 1$ was 


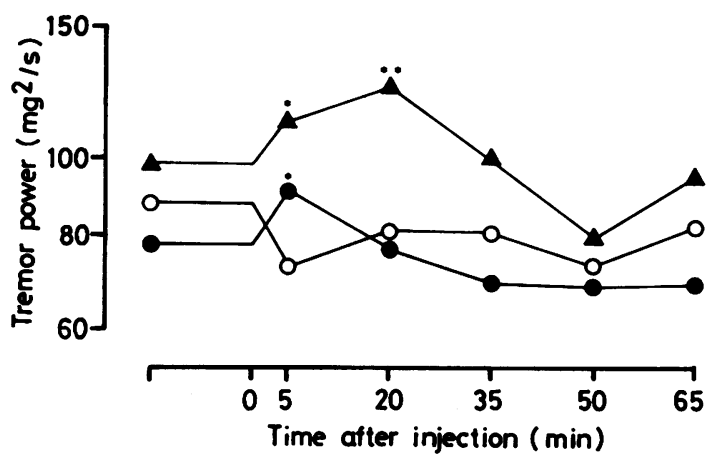

Fig 2 Effects of intravenous thymoxamine or placebo on the power of isoprenaline-enhanced physiological hand tremor. See fig 1 for explanation of symbols.

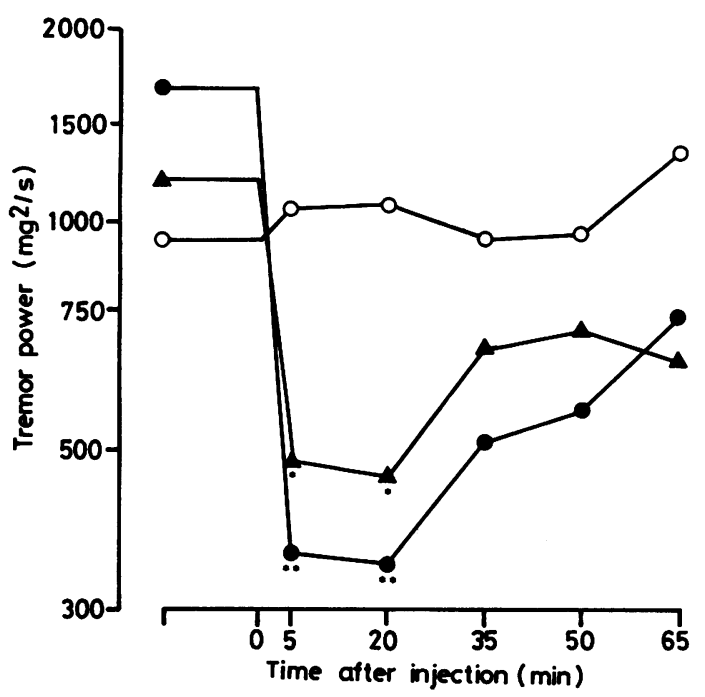

Fig 3 Effects of intravenous thymoxamine or placebo on the power of hand tremor in patients suffering from benign essential tremor. See fig 1. for explanation of symbols. Each point is the mean of 6 or, in the case of the higher thymoxamine dose, 4 measurements.

$76.6 \%$ in absolute units or $20.0 \%$ in logarithmic units, while after $\mathrm{T} 2$ administration, the maximum reduction was $53.4 \%$ or $10.9 \%$ in the different units. The values after $\mathrm{T} 1$ and $\mathrm{T} 2$ were not significantly different at any time point $(p>0.05)$. It should be noted, however, that the numerically smaller effect seen after T2 could have been due to the fact that the two patients who failed to complete the study did not receive this treatment and they were amongst the best responders to $\mathrm{T} 1$.

There was a significant negative correlation between the maximum percentage reduction in essential tremor power produced by $\mathrm{T} 1$ and the mean pre-treatment peak frequency of tremor $(\mathrm{r}=-0.86$, $\mathrm{p}<0.05)$. However, there was no significant correlation between percentage reduction in tremor power and pre-treatment tremor power $(\mathrm{r}=0.58, \mathrm{p}$ $>0 \cdot 2$ ).

\section{Discussion}

The study has demonstrated significant but differential responses by three distinct types of tremor to intravenous doses of thymoxamine. No attempt was made to age-match the normal and patient groups. Thus, the conclusions refer specifically to physiological and isoprenaline-induced tremor in a young adult group and essential tremor in a more elderly population with ages characteristic for the disease. All types of tremor showed a high level of intersubject variability. The cross-over design nevertheless allowed demonstration of the drug effects against this background of stable between-subject differences. That significant differences were evident from the small numbers of subjects studied, highlights the consistent and, in the case of essential tremor, the dramatic nature of the drug responses.

The changes in blood pressure and heart rate observed in both patients and healthy subjects following thymoxamine treatment demonstrate that the doses of thymoxamine given produced $a \propto$ significant degree of alpha-adrenoceptor blockade. The observed changes were consistent with the known effect of thymoxamine on vascular alphaadrenoceptors, the tachycardia being a reflex attempt to compensate for the sudden fall in blood pressure.

Thymoxamine significantly suppressed essential tremor in this study, confirming the report of Mai and Olsen. ${ }^{10}$ The short duration of the effect is consistent with the drug's plasma half-life of 2.5 min. $^{\text {is }}$ The tremorolytic effect of thymoxamine is most likely to have been exerted in the central nervous system (CNS). To suggest that it was exerted outside the CNS would require postulating the existence of peripheral tremorogenic alpha receptors in essential tremor patients but not in healthy subjects where alpha stimulation does not increase tremor. ${ }^{4}$ This is most unlikely.

Whether the action of thymoxamine on essential tremor was exerted in the spinal cord or at a higher level is not clear from this study. It could well be due to blockade of abnormally high noradrenergic facilitation of the gamma loop servomechanism of the stretch reflex arc. ${ }^{12}$ Further studies are necessary to investigate this possibility. However, the results indicate that central adrenergic mechanisms are involved in the pathophysiology of essential tremor.

In contrast with its effect on essential tremor, 
thymoxamine caused significant increases in physiological and isoprenaline-induced tremors. The mechanism(s) underlying this effect are not entirely clear, but could be due to effects in the periphery or in the CNS. Two possible peripheral mechanisms are that the increase in tremor was due either to an increase in the ballistocardiac impulse or to an increase in circulating adrenaline. It is not known to what extent thymoxamine-induced tachycardia could have increased ballistocardiographic activity and hence contributed to the observed increase in tremor, though the ballistocardiac impulse is thought to be responsible for only about $10 \%$ of normal postural tremor. ${ }^{16}$ To explore this point further, six healthy subjects were given 2 minutes exposure to sublingual glyceryl trinitrate tablets (1 mg BP) and physiological tremor was recorded before and 15 minutes after drug administration. Heart rate increases similar to those after thymoxamine were obtained but there was no significant effect on tremor $(p>0.05)$. It seems reasonable to extrapolate from this to say that the tachycardia seen with thymoxamine is unlikely to have made a significant contribution to the observed increase in physiological and isoprenaline-induced tremor. A similar argument can be made against the suggestion that the effect could have been due to an increase in circulating adrenaline induced by the hypotensive stress. Nevertheless, the possibility of some contribution from these mechanisms to the tremorogenic effect cannot be completely excluded.

Further possible peripheral mechanisms for the tremorogenic action of thymoxamine are the effects of vasodilation and of blocking the alphaadrenoceptors on motor nerve terminals. Vasodilation, produced by alpha blockade in skeletal muscle vasculature, is unlikely to cause tremor since vasodilators given intra-arterially do not increase physiological tremor. ${ }^{4}$ Stimulation of the alpha receptors on motor nerve terminals increases release of acetylcholine, causing facilitation of neuromuscular transmission. ${ }^{17}{ }^{18}$ Blockade of these receptors is unlikely to cause significant blockade of neuromuscular transmission which in any case is not associated with production of tremor.

The remaining and more likely explanation seems to be that the tremor-enhancing effect of thymoxamine seen in the healthy subjects was exerted in the central nervous system. The exact mechanism by which this could have been brought about, however, requires further investigation.

The differential effect of thymoxamine on essential and physiological tremor argues strongly against a similar pathophysiology for the two forms of tremor. Isoprenaline-enhanced physiological tremor cannot, therefore, be regarded as a good model of essential tremor. Furthermore, thymoxamine may be a useful pharmacological tool for the differential diagnosis between essential tremor and exaggerated physiological tremor, which may not be possible on clinical grounds alone.

The suppressive action of thymoxamine on essential tremor suggests that alpha blockers may be a useful therapy for essential tremor. Since thymoxamine is not well absorbed orally, trials with orally active alpha blockers are required to investigate this possibility. Alternatively, combined alpha-beta blockers, such as labetalol or medroxal, may prove useful in essential tremor. The negative correlation between peak frequency of tremor and percentage reduction in tremor power produced by thymoxamine suggests that patients with low frequency tremors might respond better to alpha blockade. It is interesting to note that in their studies with propranolol, Calzetti and co-workers ${ }^{19}$ also found that patients with low frequency tremors responded better to propranolol. The therapeutic and pathophysiological implications of these findings require further exploration.

We thank the following neurologists for allowing us to study patients under their care: Drs JGG Graham, CEC Wells, DAS Compston, I McQueen and NM Milligan.

\section{References}

' Halliday AM, Redfearn JWT. Finger tremor in tabetic patients and its bearing on the mechanism producing the rhythm of physiological tremor. J Neurol Neurosurg Psychiatry 1958;21:101-8.

${ }^{2}$ Lippold OCJ. Oscillations in the stretch reflex arc and the origin of the rhythmical 8-12 c/s component of physiological tremor. J Physiol (London) 1970;206: 359-82.

${ }^{3}$ Hagbarth K-E, Young RR. Participation of the stretch reflex in human physiological tremor. Brain 1979;102:509-26.

4 Marsden CD, Foley TH, Owen DAL, McAllister RG. Peripheral $\beta$-adrenoceptors concerned with tremor. Clin Sci Mol Med 1967;33:53-65.

5 Perucca E, Pickles H, Richens A. Effects of atenolol, metoprolol and propranolol on isoproterenol-induced tremor and tachycardia in normal subjects. Clin Pharm Ther 1981;29:425-33.

- Arnold JMO, Johnson GD, Harron DWG, Shanks RG, McDevitt DG. The effect of 118551 on isoprenalineinduced $\beta$-adrenoceptor responses in man. $\mathrm{Br} J$ Clin Pharmacol 1983;15:133-4P.

${ }^{7}$ Marshall J. Observations on essential tremor. J Neurol Neurosurg Psychiatry 1962;25:122-5.

${ }^{8}$ Warren JB, O'Brien M, Dalton N, Turner CT. Sympathetic activity in benign familial tremor. Lancet 1984;i:461-2.

' Growdon JH, Shahani BT, Young RR. The effect of 
alcohol on essential tremor. Neurology (Minneap) 1975;25:259-62.

${ }^{10}$ Mai J, Olsen RB. Depression of essential tremor by alpha-adrenergic blockade. J Neurol Neurosurg Psychiatry 1981;44:1171.

" Ellaway PH, Pascoe JE. Noradrenaline as a transmitter in the spinal cord. J Physiol (London) 1968;197:8$10 \mathrm{P}$.

12 Phillips SJ, Richens A, Shand DG. Adrenergic control of tendon jerk reflexes in man. $\mathrm{Br} J$ Pharmacol 1973;47:595-605.

${ }^{13}$ White CB, Richens A. Alpha-adrenoceptor blocking drugs, pressor responses to noradrenaline and the ankle jerk in man. Br J Clin Pharmacol 1974;1:223-7.

14 Wilson JF, Marshall RW, Dunstan FDJ, Richens A. A microcomputer based technique for the assessment of hand tremor in man. Br J Clin Pharmacol 1983;15:158P.
${ }^{15}$ Griffin JP, Kamburoff PL, Prime FJ, Arbab AG. Thymoxamine and airway obstruction. Lancet 1972;: 1288.

${ }^{16}$ Marsden CD, Meadows JC, Lange GW, Watson RS. The role of the ballistocardiac impulse in the genesis of physiological tremor. Brain 1969;92:647-62.

17 Bowman WC, Raper C. Adrenotropic receptors in skeletal muscle. Ann N Y Acad Sci 1967; 139:741-53.

${ }_{18}$ Malta E, McPherson GA, Raper C. Comparison of prejunctional alpha-adrenoceptors at the neuromuscular junction with vascular post-junctional alphaadrenoceptors in cat skeletal muscle. Br J Pharmacol 1979;65:249-56.

${ }^{14}$ Calzetti S, Findley LJ, Perucca E, Richens A. The response of essential tremor to propranolol: evaluation of clinical variables governing its efficacy on prolonged administration. J Neurol Neurosurg Psychiatry 1983;46:393-8. 